\title{
Ciclos de vida y componentes de la aptitud de Drosophila inca y D. yangana (Diptera, Drosophilidae)
}

\author{
Ana Beatriz Mafla M. \\ Laboratorio de Genética Evolutiva, Escuela de Ciencias Biológicas, Pontificia Universidad Católica del Ecuador, Apartado 17-01 \\ 2184, Quito, Ecuador. (amafla @ puce.edu.ec)
}

\begin{abstract}
Life cycles and fitness components of Drosophila inca and D. yangana (Diptera, Drosophilidae). The life cycles of Drosophila inca Dobzhansky \& Pavan, 1943 and D. yangana Rafael \& Vela, 2003 are described and proved to be very similar; the viability observed is different since the mortality in the larval and pupal stages of $D$. yangana is higher because of inadequate feeding. The mean duration from egg to adult is 22 days at $21-23^{\circ} \mathrm{C}$ for both species.
\end{abstract}

KEYWORDS. Life cycles, fitness, inca subgroup.

RESUMEN. Se describen los ciclos de vida de Drosophila inca Dobzhansky \& Pavan, 1943 y D. yangana Rafael \& Vela, 2003 los cuales son muy similares; la viabilidad observada es diferente ya que la mortalidad en estagios larvarios y de pupación de $D$. yangana es superior debido a la alimentación inadecuada. La duración promedia desde huevo hasta adulto es 22 días a $21-23^{\circ} \mathrm{C}$ para ambas especies.

PALABRAS-CLAVE. Ciclos de vida, componentes de la aptitud, subgrupo inca.

El grupo repleta, de amplia distribución en el neotrópico, está representado en el Ecuador por una docena de especies (RAFAEL \& VELA, 2000, 2003) y en aproximadamente dos décadas de investigación de la fauna drosophilidea ecuatoriana se han descubierto tres nuevas especies que enriquecen más al grupo. Como se conoce, por contener un gran número de especies se las ha reunido en cinco subgrupos: repleta, hydei, mulleri, fasciola, mercatorum quedando ocho especies en un conjunto misceláneo, en el cual aparece Drosophila inca Dobzhansky \& Pavan, 1943 (WASSERMAN, 1982).

El subgrupo inca fue propuesto en 1989 para reunir a Drosophila inca con una nueva especie $D$. huancavilcae Rafael \& Arcos, 1989; en el año 2001 se descubre otra especie D. yangana Rafael \& Vela, 2003, que por sus características morfológicas parece tener afinidad con D. inca y D. huancavilcae (RAFAEL \& ARCOS, 1989; Rafael \& Vela, 2003). De modo que, al momento, este subgrupo comprende tres especies, $D$. inca, $D$. huancavilcae y D. yangana.

Por lo tanto, con el objeto de caracterizar a las especies de este subgrupo del grupo repleta, se estudió el ciclo biológico de $D$. inca y el de $D$. yangana; se describen las cualidades de estas dos especies criadas y observadas en condiciones artificiales; tomando en consideración como parámetros de aptitud: la fertilidad, la viabilidad, la velocidad de desarrollo y la madurez de los adultos.

La comparación, entre especies relacionadas, puede sacar a flote las diferencias que, históricamente, se constituyeron en fuerzas centrífugas conducentes a la especiación (MAYR, 1968); por ello consideramos que el análisis de los ciclos de vida de $D$. inca y $D$. yangana puede contribuir a esclarecer el grado de relación entre las especies reunidas en el subgrupo inca.

\section{MATERIAL Y MÉTODOS}

En este estudio se usaron tres isolíneas de $D$. yangana y tres de $D$. inca fundadas a partir de hembras recolectadas en la naturaleza durante marzo del 2002 en la localidad andina de Yangana, provincia de Loja, Ecuador $\left(4^{\circ} 23^{\prime} \mathrm{S}, 79^{\circ} 11^{\prime} \mathrm{W}\right)$. Las seis isolíneas fueron multiplicadas en el laboratorio en tubos de ensayo con el medio de cultivo descrito por RAFAEL et al. (2000) enriquecido con un trozo de la fruta madura de tuna (Opuntia soederstromiana); la temperatura ambiental se mantuvo en un estrecho margen de $21^{\circ} \mathrm{C}$ a $23^{\circ} \mathrm{C}$, con períodos de luz: oscuridad (12h:12h). Debido al hecho de que se suplementaba el medio con un trozo de la fruta fresca, la contaminación con hongos fue frecuente y obligó a cambios de papel y fruta fresca dos veces por semana.

Se midieron diez huevos de $D$. inca; para ello se usó un estéreo microscopio y un portaobjetos milimetrado al cual se le sobreponía una laminilla con el objeto en posición dorsal y en seco; se midió la longitud desde el extremo anterior hasta el extremo posterior y la porción central más ancha. No se cuenta con datos de $D$. yangana por que son muy vulnerables a la manipulación y se dio prioridad a la observación del desarrollo y multiplicación de individuos.

Desde el 17 de septiembre hasta el 31 de octubre del 2002 se hicieron observaciones diarias pero, a medida que el número de siembras aumentaba, los registros fueron espaciándose cada dos ó tres días; anotándose las fechas de aparecimiento de huevos, y de cada fase de desarrollo hasta el momento de emergencia de adultos. De D. yangana se alcanzó un número de 309 siembras: 105 correspondientes a la isolínea uno, 101 a la isolínea dos y 103 a la isolínea tres; para $D$. inca se hicieron 313 siembras: 105 de la isolínea uno, 106 de la dos y 102 de la tres, dando un total de 622 tubos con siembras hasta el 28 de febrero del 2003. 
En la descripción del ciclo se hace referencia al modo (moda o número modal) como un parámetro más adecuado; pero también se dan los valores medios y las desviaciones estándar en días de duración de cada fase de desarrollo. Los componentes de la aptitud presentados son: la fertilidad definida simplemente como el número de descendientes producido (MATHER, 1983); tal valor se expresa en número de tubos conteniendo huevos por número de tubos sembrados y se transforma a porcentaje. La viabilidad expresada en número de tubos en los que emergieron adultos por el número de tubos que contenían huevos e igualmente transformada en porcentaje; la madurez del adulto: tiempo promedio, en días, que demora el adulto para cruzarse y dejar descendencia y la velocidad de desarrollo expresada en promedio de días de duración desde la eclosión de la larva hasta la emergencia del imago.

En el análisis de varianza se aplicó la prueba $\mathrm{F}$ de Snedecor para probar la hipótesis nula de que las seis isolíneas de las dos especies del subgrupo inca tienen la misma duración en cada una de las fases del ciclo.

\section{RESULTADOS Y DISCUSIÓN}

Los huevos de $D$. inca tienen 0,6 mm de longitud y $0,2 \mathrm{~mm}$ en la parte central más ancha; presentan cuatro filamentos de 0,5 mm de longitud: dos nacen del extremo anterodorsal y dos emergen aproximadamente a un tercio de la longitud en la superficie ventral. Los huevos de $D$. yangana son más pequeños. Esta primera fase tiene una duración de tres días (número modal). Si tomamos como valor referencial al promedio parecería que $D$. inca es más rápida que $D$. yangana en la emergencia del primer estadio larvario: 2,4 días frente a 3,3 (tabs. I, II).

Al segundo o tercer día de la ovoposición emerge una pequeña larva de cerca de $1 \mathrm{~mm}$ de largo y tiene dos mudas; las dos primeras fases larvarias presentan un número modal de tres días de duración en las dos especies. Pero, tomando en cuenta los valores promedios, hay que notar que las larvas de primer estadio de $D$. inca parecerían ser algo más activas y pasar al segundo estadio

Tabla I. Duración en días de cada fase de desarrollo en Drosophila inca Dobzhansky \& Pavan, 1943 (EST, estadio; n, número de tubos; s, desviación estándar de la muestra; $\sigma$, desviación de la población).

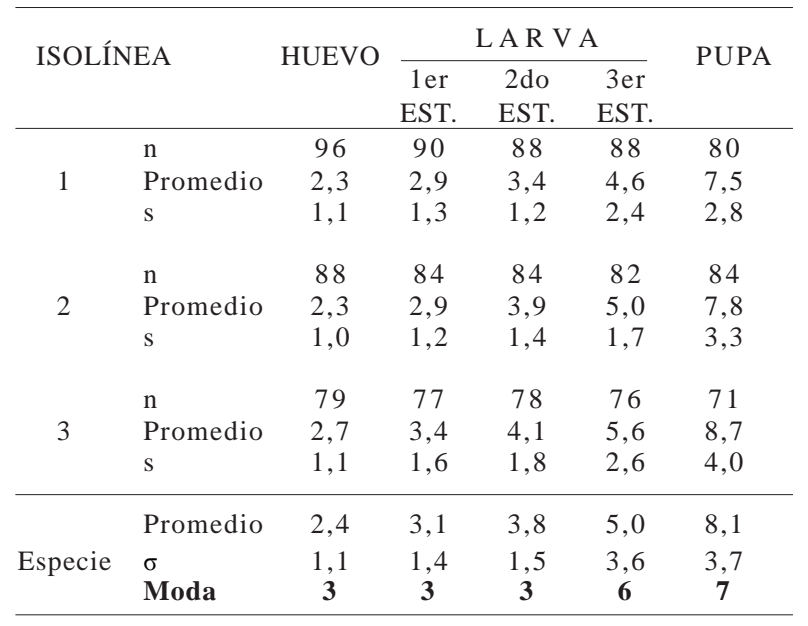

Tabla II. Duración en días de cada fase de desarrollo en Drosophila yangana Rafael \& Vela, 2003 (EST, estadio; n, número de tubos; s, desviación estándar de la muestra; $\sigma$, desviación de la población).

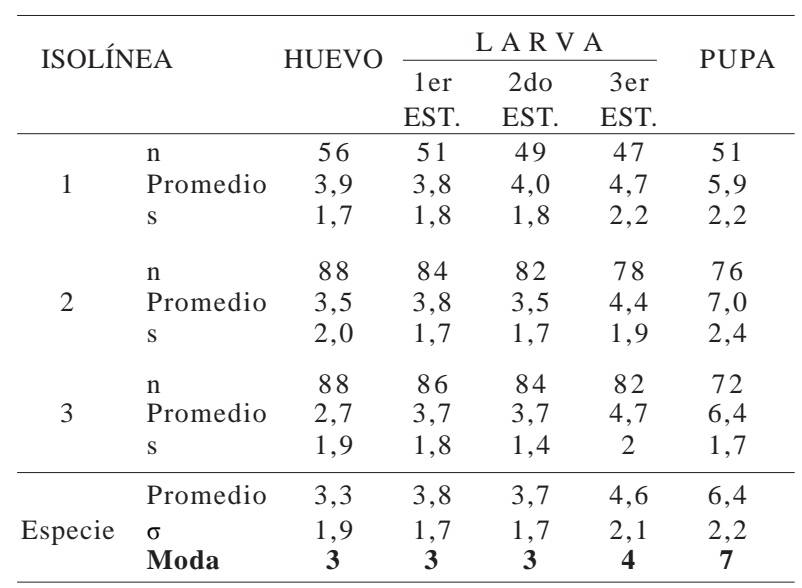

algo más temprano: 3,1 días frente a 3,8 días que demoran las larvas de D. yangana.

Durante el tercer estadio larvario hay una diferencia marcada de dos días, D. yangana es más rápida para empupar con un número modal de cuatro días frente a seis que le ocupa a $D$. inca. Los valores promedio, en cambio, son semejantes: 5,0 en $D$. inca y 4,6 en $D$. yangana.

El período de pupación de siete días, número modal, otra vez resulta coincidente en las dos especies; aunque los valores promedio muestran una diferencia de 1,7 días.

La prueba de $\mathrm{F}$ arroja valores significativos para los períodos de duración de huevo, larva de primer estadio y pupa (tab. IV); esto podría estar relacionado con las exigencias particulares de cada especie que afloran como diferencias en las condiciones homogéneas de los cultivos.

Valores de los componentes de la aptitud encontrados en las tres isolíneas de cada especie (tab. III): las cifras promedio de fertilidad son $88,7 \%$ para $D$. yangana y $92,7 \%$ para $D$. inca; hay una diferencia del $4 \%$ entre las dos especies pero esto no parece ser biológicamente significativo porque entre las isolíneas 1 y 2 de D. yangana hay una diferencia del 8,3\%; y entre las isolíneas 1 y 2 de $D$. inca del 5,6\%. Como los adultos sembrados en los 622 tubos fueron individuos recién eclosionados, la explicación para que no aparecieran huevos en todas las siembras puede ser que: dichos adultos no sean hábiles para aparearse; o que los gametos no estuvieran todavía maduros o que la contaminación de los cultivos impidiese la ovoposición; cualquiera sea la razón, lo que se observa es mayor variación entre isolíneas de la misma especie que entre especies.

En cuanto a la viabilidad hay una diferencia de 5,4 en los valores porcentuales: D. yangana presenta un $81,2 \%$ en tanto que el valor es de $86,6 \%$ en $D$. inca. La variación entre isolíneas de la misma especie: 4,3 en $D$. yangana y 4,5 en $D$. inca aparece menor que entre especies. La mortalidad en los períodos larvario y de pupación es mayor para D. yangana; probablemente el medio de cultivo no reúna las condiciones óptimas para la crianza de esta especie. Cabe observar que cuando el suplemento de fruta fresca es descuidado las cepas de esta especie se deprimen rápidamente. 
Tabla III. Componentes de la aptitud en Drosophila inca Dobzhansky \& Pavan, 1943 y D. yangana Rafael \& Vela, 2003 (A/H, tubos en los que emergieron adultos por tubos con huevos; H/ $\mathrm{S}$, tubos con huevos por tubos con siembras); velocidad de desarrollo y la maduración de adultos en días (n, número de tubos; s, desviación estándar de la muestra; $\mu$, promedio de la población; $\sigma$, desviación de la población).

\begin{tabular}{|c|c|c|c|c|c|c|}
\hline \multirow{2}{*}{ ISOLÍNEAS } & \multicolumn{3}{|c|}{ D. inca } & \multicolumn{3}{|c|}{ D. yangana } \\
\hline & 1 & 2 & 3 & 1 & 2 & 3 \\
\hline $\mathrm{H} / \mathrm{S}$ & $100 / 105$ & $95 / 106$ & $95 / 102$ & $88 / 105$ & $93 / 101$ & $93 / 103$ \\
\hline Fertilidad & \multicolumn{3}{|c|}{$290 / 313=92,7 \%$} & \multicolumn{3}{|c|}{$274 / 309=88,7 \%$} \\
\hline $\mathrm{A} / \mathrm{H}$ & $85 / 100$ & $85 / 95$ & $81 / 95$ & $72 / 88$ & $77 / 93$ & $73 / 93$ \\
\hline Viabilidad & \multicolumn{3}{|c|}{$251 / 290=86,6 \%$} & \multicolumn{3}{|c|}{$222 / 274=81,2 \%$} \\
\hline $\mathrm{n}$ & 86 & 84 & 75 & 73 & 78 & 73 \\
\hline Promedio & 20,8 & 21,9 & 24,1 & 22,2 & 21,8 & 21,0 \\
\hline s & 5,1 & 4,0 & 4,9 & 4,2 & 4,2 & 3,9 \\
\hline \multicolumn{2}{|c|}{$\begin{array}{l}\text { Velocidad de } \\
\text { desarrolo }(\mu \pm \sigma)\end{array}$} & \multicolumn{2}{|c|}{$22,3 \pm 4,7$} & \multicolumn{3}{|c|}{$21,6 \pm 4,1$} \\
\hline $\mathrm{n}$ & 100 & 88 & 84 & 77 & 90 & 90 \\
\hline Promedio & 5,3 & 5,9 & 6,0 & 5,9 & 5,6 & 5,8 \\
\hline $\mathrm{s}$ & 2,5 & 2,1 & 2,2 & 2,1 & 2,0 & 2,2 \\
\hline $\begin{array}{l}\text { Maduración } \\
\text { adulto }(\mu \pm \sigma)\end{array}$ & \multicolumn{3}{|c|}{$5,7 \pm 2,3$} & \multicolumn{3}{|c|}{$5,8 \pm 2,1$} \\
\hline
\end{tabular}

Con respecto a la velocidad de desarrollo o tiempo de generación los números modales para las dos especies son de 21 días; los promedios también son muy parecidos: 21,6 y 22,3 días es la duración total del ciclo en $D$. yangana y $D$. inca, respectivamente. Por lo que en el período de observación se han registrado datos para 7 a 8 generaciones.

La maduración de adultos de unos seis días promedio, así como el rango de variación entre isolíneas también es similar en ambas especies.

Las varianzas en la velocidad de desarrollo resultan con un valor de F significativo; en tanto que para la maduración de adultos no lo son (tab. IV).

La semejanza en el período de maduración de los adultos de las dos especies nos hacen pensar en su parentesco, mientras que las diferencias que hay en: fertilidad, viabilidad y velocidad de desarrollo nos cuentan
Tabla IV. Varianzas y valores $\mathrm{F}$ de la duración de cada fase del ciclo de las dos especies de Drosophila inca y D. yangana (*, significativo a una $\mathrm{P}=0,01$ ).

\begin{tabular}{lrcc}
\hline & \multicolumn{2}{c}{ Varianzas } & \multirow{2}{*}{$\mathrm{F}$} \\
\cline { 2 - 3 } & D. inca & D. yangana & \\
\hline Huevo & 1,21 & 3,77 & $3,1157^{*}$ \\
1er estadio & 2,04 & 3,04 & $1,4901^{*}$ \\
2do estadio & 2,38 & 2,79 & 1,1722 \\
3er estadio & 5,52 & 4,23 & 1,3049 \\
Pupa & 13,80 & 4,89 & $2,822^{*}$ \\
Velocidad de desarrollo & 24,37 & 17,25 & $1,4127^{*}$ \\
Maduración de adultos & 5,29 & 4,41 & 1,1995 \\
\hline
\end{tabular}

sobre las vías alternativas que habrían conducido a los dos linajes a diferenciar estrategias que les permita la actual coexistencia; estos datos, por tanto, corroboran la propuesta de reunirlas en el subgrupo inca.

Agradecimientos. A mis colegas Violeta Rafael y Varsovia Cevallos por sus valiosos comentarios y sugerencias. El presente trabajo es parte del proyecto: "Caracterización de nuevas especies ecuatorianas de Drosophila", financiado por la Pontificia Universidad Católica del Ecuador.

\section{REFERENCIAS BIBLIOGRÁFICAS}

Mather, K. 1983. Response to Selection. In: Ashburner, M.; Carson, H. L. \& Thompson, J. N., Jr. eds. The Genetics and Biology of Drosophila. London, Academic. v. 3c, p. 155221.

Mayr, E. 1968. Especies Animales y Evolución. Barcelona, Universidad de Chile y Ediciones Ariel. 808 p.

Rafael, V. \& Arcos, G. 1989. Subgrupo inca, un nuevo subgrupo del grupo repleta, con descripción de Drosophila huancavilcae nov. sp. (Diptera, Drosophilidae). Evolución Biológica, 3(3): 233-243.

RafaEl, V. \& Vela, D. 2000. Drosophila distribution in Ecuador. Drosophila Information Service, 83:85-88.

2003. Drosophila yangana sp. nov. un nuevo miembro del grupo repleta, subgrupo inca (Diptera: Drosophilidae). Revista de la Pontificia Universidad Católica del Ecuador, 71:129-139.

Rafael, V.; Arcos, G. \& Arcos, L. 2000. Ecología y distribución del género Drosophila en Guayllabamba y El Quinche, Provincia de Pichincha-Ecuador. Revista de la Pontificia Universidad Católica del Ecuador, Quito, 65:130-155.

Wasserman, M. 1982. Evolution of the repleta Group. In: Ashburner, M.; Carson, H. L. \& Thompson, J. N., Jr. eds. The Genetics and Biology of Drosophila. London, Academic. v. 3b, p. 61-139.

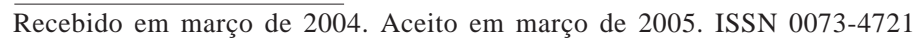

Artigo disponível em: www.scielo.br/isz 